\title{
OBSERVATIONAL ASPECTS OF STELLAR EVOLUTION IN THE UPPER HRD
}

\author{
CLAUS LEITHERER \\ Space Telescope Science Institute, 3700 San Martin Drive, Baltimore, MD \\ 21218. Affiliated with the Astrophysics Division of the Space Science Depart- \\ ment of the European Space Agency
}

\section{Introduction: Wolf-Rayet Stars and Their Relation to Massive Stars}

Observational evidence strongly favors an evolutionary relation between Wolf-Rayet (WR) stars and stars with masses above $\sim 10 M_{\odot}$. The galactic distribution of WR stars closely resembles the distribution of $O$ stars both in the $z$-direction as well as in the spiral-arm pattern (Hidayat, Admiranto, and van der Hucht 1984, van der Hucht et al. 1988, Conti and Vacca 1990). Lundström and Stenholm (1984) found that the surface density of WR stars increases with decreasing distance from $O B$ associations as well as from young open clusters. The fraction of WR stars in clusters turns out to be about the same as that of $\mathrm{O}$ stars. A large number of extragalactic WR stars is situated in giant $\mathrm{H}$ II regions, which also contain high numbers of very massive $O$ stars (see, e.g., Walborn 1990 for 30 Doradus; Drissen, Moffat, and Shara 1990 for M33). Many WR stars are members of binary systems with well established parameters so that their masses can be determined directly. In several cases, WR-star masses of $M \gtrsim 10 M_{\odot}$ have been derived (Massey 1981, St.-Louis et al. 1987, Schulte-Ladbeck 1989).

Furthermore, there is overwhelming observational support for the assumption that WR stars are in a later evolutionary stage than $O$ stars (see Lamers et al. 1990). Abundance analyses of WR stars are consistent with a scenario that we are seeing material processed by the CNO cycle and by He burning (Hillier 1990). WR stars are evolved objects. The spectral appearance of some WNL stars closely resembles highly evolved objects, such as Luminous Blue Variables (Stahl 1986, Walborn 1989). The observed mass-luminosity relation agrees with the assumption that WR stars are highly evolved objects, rather than pre-main-sequence stars. Finally, evolved descendants of massive $O$ stars are expected to be luminous and should easily be detected. If WR stars are not descendants of massive stars, it is hard to understand why such descendants have not yet been discovered (Lamers et al. 1990).

It should be mentioned that an alternative interpretation has been given by Underhill (this meeting), who suggests WR stars are predecessors of $O$ stars on their way to the main sequence. Considering all the observational evidence summarized above, $I$ will assume in this review that WR stars are evolved descendants of previously massive stars.

Our theoretical modeling of massive-star evolution becomes more and more uncertain the further a star has evolved from the main sequence. Obviously, a thorough understanding 
of the pre-WR evolution of a star is required in order to understand the WR phase itself. I will review our current observational database available to study stellar evolution in the upper part of the HRD. The main emphasis will be on aspects which are of direct relevance to WR stars, such as channels which lead to the formation of WR stars, the mass regime of WR predecessors, the location of WR predecessors in the HRD, and consequences of different chemical composition on the relation between WR stars and their progenitors.

\section{The Upper HRD - an Overview}

The most massive stars are also the most luminous stars - even visually since they follow a mass-luminosity relation of $L \sim M^{\alpha}$, with $\alpha>1$ (Maeder 1987). Complete samples for the population of massive stars in the solar neighborhood with $M \gtrsim 10 M_{\odot}$ have been published by a variety of authors, most recently by Blaha and Humphreys (1989). Figure 1 reproduces their observed HRD of all known massive stars which are members of clusters or associations within $3 \mathrm{kpc}$ of the sun. The schematic location of individual WR subgroups taken from Schmutz, Hamann, and Wessolowski (1989) is indicated. WR stars roughly coincide in their location with main sequence $O$ stars - except for WNE-types, which are slightly hotter. I also included the positions of several well-known Luminous Blue Variables (LBV's, Humphreys 1989).

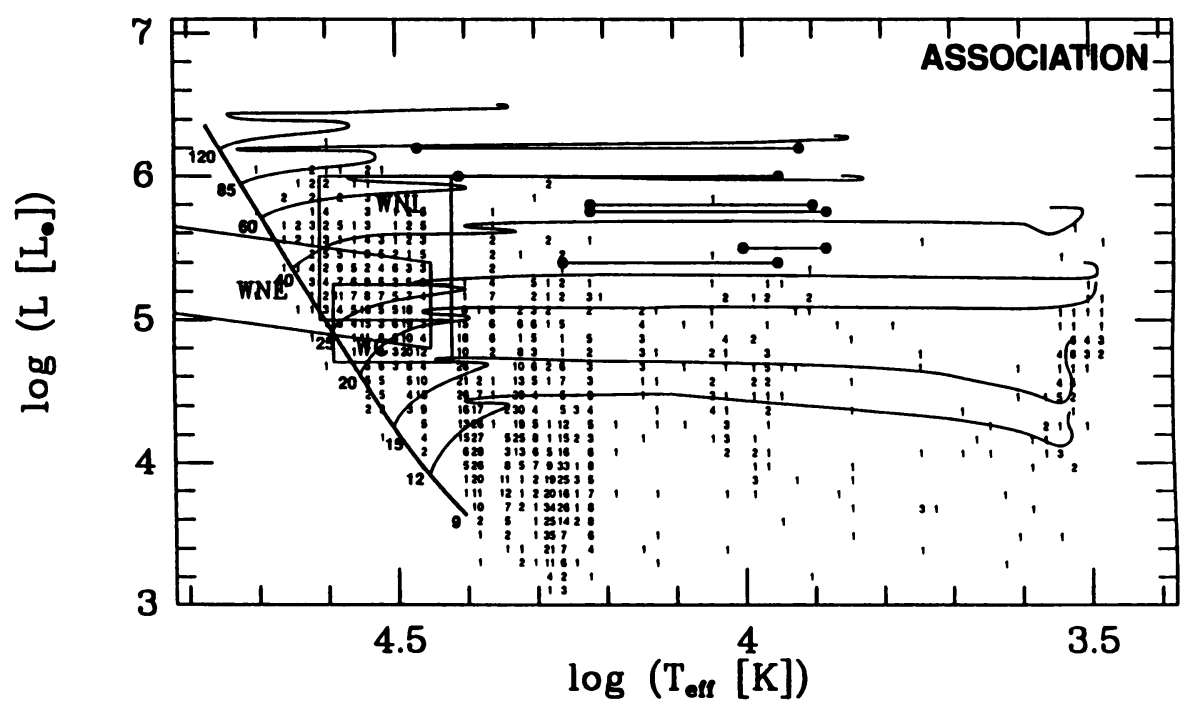

Figure 1. Observed HRD of the solar neighborhood (Blaha and Humphreys 1989). Numbers indicate star densities in the HRD. Solid dots denote the location of LBV's. WR stars are indicated schematically by boxes.

Blaha and Humphreys confirm - using more refined observational data - an important result first detected by Humphreys and Davidson (1979). The most luminous blue stars are about a factor of 6 more luminous (and thus more massive) than the most luminous 
red stars. This difference in luminosity defines a boundary in the HRD parameterized by Garmany, Conti, and Massey (1987) and Humphreys (1987). The importance of this boundary is obvious: Stars evolve from the main sequence towards cooler temperatures at roughly constant luminosity (e.g. Maeder and Meynet 1988). The observed absence of stars to the right of the boundary may mean that stellar evolution proceeds so fast that the probability of observing red stars with $M \gtrsim 50 M_{\odot}$ is very low. Langer and El Eid (1986) computed evolutionary models for a $100 M_{\odot}$ star evolving into a red supergiant (RSG) and then back into a WR star. They find that the star should spend $10^{4}-10^{5} \mathrm{yr}$ in the RSG phase. Although this lifetime is rather uncertain (e.g. due to the mass loss in the RSG phase), one would expect that luminous RSG's with $L \approx 10^{6} L_{\odot}$ should be detected if they existed.

Alternatively, the observed boundary in the HRD can be understood if massive stars with $M \gtrsim 50 M_{\odot}$ evolve from the main sequence via the blue supergiant (BSG) phase into WR stars. Maeder (1983) demonstrated that such an evolutionary scenario is possible if a phase of strong mass loss occurs during post-main-sequence evolution in order to remove hydrogen-rich layers from the stellar surface. The star can then evolve into the WR regime without entering the RSG phase. Evidence for such a phase of high mass loss comes from observations of LBV's (Humphreys 1989). Some of these objects are located close to the boundary of the HRD (cf. Figure 1). An estimate of their lifetimes and mass-loss rates suggests that LBV's lose a significant fraction of their total mass in this part of the HRD. Hence, they have subsequently been identified with the phase of strong mass loss a priori postulated by stellar evolution models.

The following scenario emerges from the shape of the upper HRD: Stars with initial masses below about $50 M_{\odot}$ evolve from the main sequence to the RSG phase and may return back to the blue part to form a WR star, depending on how much mass can be removed from the $H$-rich envelope via mass loss. Stars above about $50 M_{\odot}$ experience mass-loss rates $(\dot{M})$ in the blue part of the HRD high enough that these stars enter the WR phase without passing through the RSG phase. Such a genetic relationship has been proposed by Maeder (1983).

\section{Properties of the Observed HRD: Clues for WR Evolution}

\subsection{THE LACK OF VERY MASSIVE STARS CLOSE TO THE ZAMS}

Close inspection of Figure 1 reveals a lack of very massive stars with $M \gtrsim 40 M_{\odot}$ close to the theoretical zero-age main sequence (ZAMS). Such an effect has also been noted by Garmany, Conti, and Chiosi (1982) on the basis of a different data set. From a comparison with theoretical isochrones one finds that the youngest known hydrogen-burning $\mathrm{O}$ stars have an age of $1-210^{6} \mathrm{yr}$ (see, e.g., Chiosi 1986), so that $\sim 20 \%$ of the entire main sequence lifetime is not observed.

Wood and Churchwell (1989) found that $10-20 \%$ of all $O$ stars in the solar neighborhood (SN) are still embedded in molecular clouds. They are detectable indirectly by radiation from ultracompact $\mathrm{H}$ II regions and warm circumstellar dust surrounding the central object. Only about $10^{6} \mathrm{yr}$ after the onset of H-burning circumstellar gas and dust become transparent and the star becomes visible. This seems to be a plausible explanation for the observed lack of very massive ZAMS stars in the HRD.

The fact that we may underestimate the true number of $O$ stars in the SN by $\sim 20 \%$ is of 
immediate relevance for evolutionary models of WR stars. An important - because easily (!) observable - parameter predicted by models is the number ratio of WR/O stars (see below). The ratio previously derived from observations may have to be revised downward by $\sim 20 \%$.

\subsection{THE OBSERVED MAIN-SEQUENCE WIDTH}

The observed width of the main sequence provides another important parameter for the evolution on the main sequence and for post-main-sequence evolution. Constraints for the amount of overshooting can be derived, which in turn governs the formation of supergiants and WR stars. Bertelli, Bressan, and Chiosi (1984) determined the main sequence width by counting stellar number densities across a synthetic HRD of the SN. Alternatively, Mermilliod and Maeder (1986) fitted theoretical isochrones to the observed color-magnitude diagrams of individual clusters. Both studies conclude that the main sequence extends to early- or even late-B stars in the upper HRD so that many OB supergiants are still core-hydrogen burning. Maeder and Meynet (1987) demonstrated that the observed shape of the main sequence can be used to constrain the amount of mass loss and overshooting in evolutionary models.

Bertelli, Bressan, and Chiosi (1984) emphasized the strong sensitivity of the mainsequence width predicted by evolution models on the adopted opacities. Since theoretical opacities are subject to uncertainties, this point should be kept in mind.

Recently, Garmany (1990) reinvestigated the observed main-sequence width in clusters and associations. Preliminary results seem to indicate that earlier studies tend to overestimate the main sequence width: if field stars are erroneously assigned cluster membership and/or if stars did not form coevally, the apparent width of the main sequence will be increased.

\subsection{THE DISTRIBUTION OF OB SUPERGIANTS IN THE HRD OF THE LMC}

With improved spectral classifications becoming available for more and more stars in the LMC, Fitzpatrick and Garmany (1990) are able to construct a complete, homogeneous upper HRD with LMC stars. Figure 2 is taken from their paper. The upper luminosity limit agrees with the results derived for the SN (cf. Figure 1). Comparison of HRD's of several Local Group galaxies covering a wide range of chemical composition reveals no significant variation of the shape of the upper boundary (Blaha and Humphreys 1989). Differences in the luminosities of the most luminous blue stars found in these galaxies can be accounted for by statistical effects (Kennicutt 1983, Schild and Maeder 1983). This does not exclude that there may be differences in stellar evolution and/or the initial mass function from galaxy to galaxy. However, stellar statistics is too poor to answer this question on the basis of the upper limit of the HRD.

Strikingly, there is a pronounced discontinuity in the density distribution among blue supergiants. Note that this discontinuity is marginally discernible also in the HRD of the SN but tends to be "washed out" due to uncertainties in the distances of galactic stars. Such a discontinuity can be understood if red supergiants with initial masses of $10 M_{\odot}<M<40 M_{\odot}$ undergo a blueward loop shortly before core-helium exhaustion (see Chiosi and Summa 1970, Langer, El Eid, and Baraffe 1990). Note that in these models the stars situated to the left of the discontinuity in Figure 2 are not immediate progenitors of WR stars but will return back to the RSG stage until core-helium exhaustion. On the other 
hand, a recent analysis of the Ofpe/WN star R84 (Schmutz et al. 1990) resulted in stellar parameters which place this star right on the density enhancement in the HRD $(\log L=5.7$, $\log T_{\text {eff }}=4.45$ ). Despite the same location in the HRD as the stars supposedly on the blue loop, R84 shows spectral characteristics of a late WN star, which may indicate a different evolutionary phase for this star.

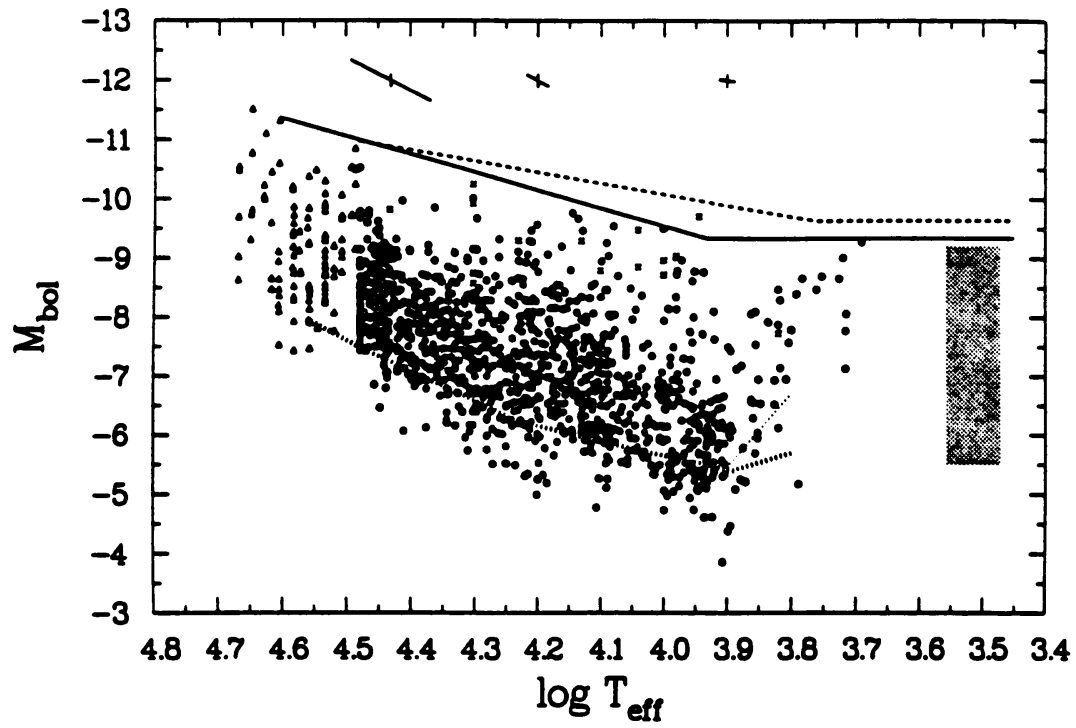

Figure 2. Upper HRD of the LMC published by Fitzpatrick and Garmany (1990). Note the pronounced discontinuity in star density among blue supergiants.

\subsection{OBSERVED EVIDENCE FOR CNO- AND HE-ANOMALIES IN EVOLVED STARS}

The hypothesis outlined above that many OB stars are actually evolved further than naively deduced from their location in the HRD is further substantiated by observations of CNO processed material in some of these stars. Walborn (1976) first drew attention to the morphological group of OBN/OBC stars, which show nitrogen- and carbon-line strengths different from what is observed in other stars with the same spectral type. It has been suggested (Walborn 1988) that the majority of "normal" B supergiants shows processed material whereas OBC objects are the rare, unevolved objects. Further support for the presence of chemically evolved objects close to the main sequence comes from spectral analyses performed by Kudritzki, Simon, and Hamann (1983) and Bohannan al. (1986) who derived significant He overabundances for several OB stars.

Clearly, the above cases demonstrate a weakness inherent in the HRD as a tool to study stellar evolution. Evolutionary effects are more complex than can be described in a twoparameter space limited to $L(t)$ and $T_{\text {eff }}(t)$. The variation of $M$ and the chemical composition on the stellar surface with lifetime may profoundly change the spectral appearance, as in the case of a WR star. On the other hand, these effects may be subtle enough that stars in very different evolutionary phase at the some location of the HRD may be hard to distinguish, as may be the case with certain blue supergiants. 


\subsection{THE LBV - WR CONNECTION}

Luminous Blue Variables are an important link between massive stars still in their corehydrogen phases and WR stars. They exhibit spectral variations on time-scales of years to decades. At one time they closely resemble other blue supergiants in the same part of the HRD and at the other they are indistinguishable in their spectral morphology from certain WN9 stars (Stahl 1986). The total bolometric luminosity of LBV's remains constant even if $T_{\text {eff }}$ varies between $\sim 30,000 K$ and $\sim 8,000 K$ (cf. Figure 1 ). Therefore, $L$ can be determined with relatively high accuracy when these objects are in their low-temperature phase - in contrast to WNE- or WC-stars, where high (and uncertain) bolometric corrections must be applied.

Since the wind characteristics of LBV's are not as pronounced as those of WR stars, their photospheres are much better accessible for a detailed analysis (e.g., Kudritzki et al. 1989, Leitherer et al. 1989). The most important results of such studies are a relatively low mass and He enrichment at the stellar surface. Under the assumption that the stellar winds in these objects are driven by radiation pressure, the low wind velocities (as compared with other supergiants at the same position in the HRD) observed in LBV's are an immediate consequence of the low surface escape velocity, and thus of the low mass. It is interesting to note that the WNL stars analyzed by Schmutz, Hamann, and Wessolowski (1989) have the lowest terminal velocities $\left(900-1200 \mathrm{~km} \mathrm{~s}^{-1}\right)$ among all WR stars of their sample. This may also indicate the close evolutionary relationship between (some ?) LBV's and WNL stars.

Comparison with evolutionary models suggests that LBV's with ZAMS masses below $\sim 40 M_{\odot}$ may be post-red supergiants on their way to becoming WR stars. Alternatively, stars in this mass range could already encounter the LBV phase immediately after the BSG phase and never enter the RSG phase. If the latter is true - and if LBV's are in fact progenitors of WN stars - there should be no correspondence between the populations of WN stars and RSG's above a certain threshold mass. It seems more likely, though that LBV's with $M_{Z A M S} \leqslant 40 M_{\odot}$ did pass through the RSG stage because of the presence of a dust shell discovered around R71 (Wolf and Zickgraf 1987). This dust shell may have formed when R71 was still in its RSG phase. With respect to LBV's with higher initial mass, it seems implausible that stars in this mass range ever evolve into the red part of the HRD (cf. Section 2).

\section{The Mass Regime of WR Predecessors}

The previous section addressed potential connections between massive stars in early and late stages of their evolution. I will now review what mass range of stars on the ZAMS will eventually evolve into WR stars.

Conti et al. (1983) studied the correlation of the O-star population in the SN with the WR population. They found that the WR population correlates best with the population of $O$ stars having initial masses above $\sim 40 M_{\odot}$. Doom (1987) and van der Hucht et al. (1988) readdressed the issue using a more rigorous statistical analysis and improved observational data. In agreement with Conti et al. they derive a typical progenitor mass of $M_{Z A M S} \geq 35$ $M_{\odot}$. However, some WR stars may descend from stars down to $M_{Z A M S} \approx 22 M_{\odot}$. On the average, WN stars have lower progenitor masses than have WC stars. Interestingly, by separating those WR stars which are members of binaries from their sample, van der Hucht 
et al. showed that single WR stars preferentially descend from higher mass stars than do WR stars in binaries. This agrees with what would be expected theoretically: stars with initially higher masses experience higher mass loss, which favors the formation of WR stars so that the binary channel to form WR stars becomes less important (see Maeder 1990a).

Schild and Maeder (1984) used the membership of WR stars in clusters and OB associations to determine lower cut-off masses for the formation of WR subtypes. By fitting theoretical isochrones to the observed main sequence turn-off, the mass limits of the WR progenitors can be derived. Schild and Maeder find $M_{Z A M S}(W N) \gtrsim 18 M_{\odot}$ and $M_{Z A M S}(W C)$ $\gtrsim 35 M_{\odot}$. Contrary to the results derived by van der Hucht et al., no significant difference could be detected for the progenitor masses of binary or single WR stars. Humphreys, Nichols, and Massey (1985) performed a similar type of study and derive $M_{Z A M S}(W R)$ $\gtrsim 30 M_{\odot}$, with $80 \%$ of all WR stars originating from progenitors above $50 M_{\odot}$. WN and WC stars showed no significantly different cut-off mass.

The results of these studies imply that the lower cut-off mass of WR progenitors is around $25 \pm 10 M_{\odot}$. WC stars may descend from higher-mass stars than do WN stars but the correlation is not so clear. One should be aware that we are dealing with small-number statistics since the number of $\mathrm{O}$ - and WR-stars in individual clusters and associations may be low. Also, the assumption of cluster membership or coeval star formation may not be correct in some cases.

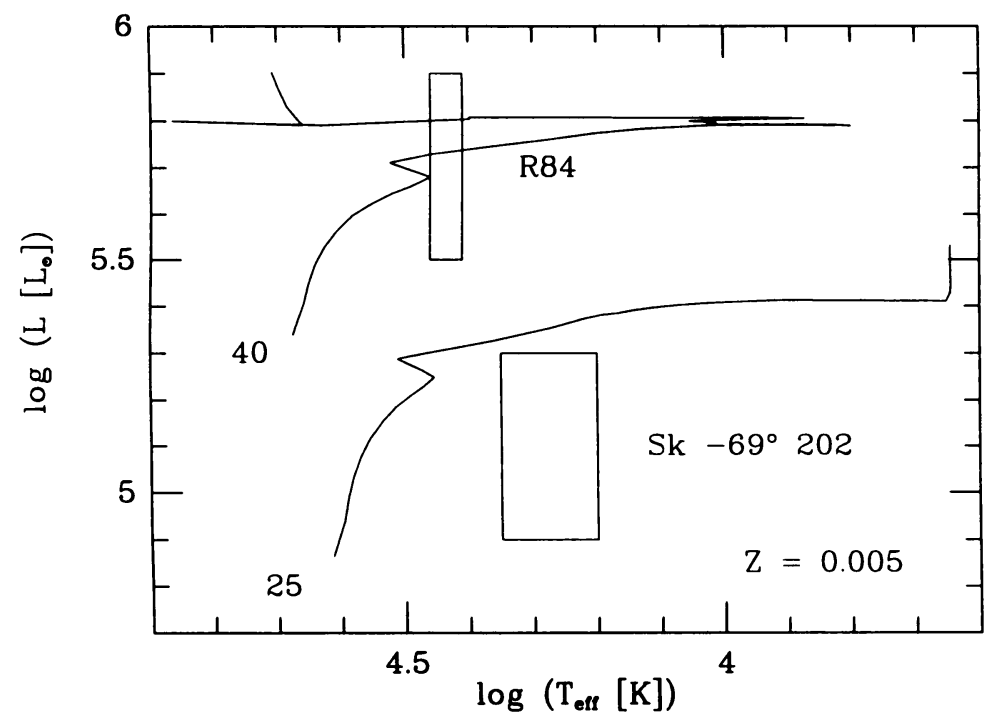

Figure 3. Location of the two LMC stars R84 and Sk $-69^{\circ} 202$ in the HRD. Evolutionary tracks for LMC abundances are from Maeder (1990b). R84 has been classified as Ofpe/WN9 whereas Sk $-69^{\circ} 202$ exploded as a supernova without entering the WR phase.

Figure 3 is a HRD giving the location of two well-known LMC stars. One star is Sk $-69^{\circ} 202$, the progenitor of SN1987A (see Arnett et al. 1989), the other is R84, an Ofpe/WN star recently analyzed by Schmutz et al. (1990). Sk $-69^{\circ} 202$ never entered the WR phase whereas R84 exhibits spectral features typical for 1 - ' n WN stars. The progenitor masses 
of both stars are relatively well-known: $20-25 M_{\odot}$ for $S k-69^{\circ} 202$ and $30-40 M_{\odot}$ for R84. Somewhere between these two mass ranges a critical mass may exist below which the channel for the formation of WR stars is closed. This argument assumes that initial mass (and possibly chemical composition, see below) is the only factor determining this channel. Presently, nothing can be said on the importance of other effects such as, e.g., stellar rotation.

\section{The Relative Numbers of WR Stars and Their Progenitors}

If WR stars are the evolved descendants of stars having main-sequence masses above $25 M_{\odot}$, a relation between the observed numbers of WR stars and their progenitors can be expected. Such a relation, like the ratio $\frac{W R}{O}=\frac{\text { NumberofWRStars }}{\text { NumberofOStars }}$, reflects the lifetimes of the star spent in each of the two individual phases. The results obtained for the SN are summarized in Figure 4 (taken from Leitherer 1990). The dots in this figure indicate the stellar number counts for O stars (Garmany, Conti, and Chiosi 1982) and WR stars (Conti et al. 1983). From this data set one finds $\frac{W R}{O}=0.12$. Theoretical predictions are indicated by the solid line in Figure 4. These models have been computed with an initial mass function and star formation rate as derived by Garmany, Conti, and Chiosi (1982) and stellar evolution models published by Maeder and Meynet (1988). Observations and model predictions agree for the relative and absolute numbers of $O$ stars. In contrast, the models produce about a factor of 2 less WR stars than are observed.

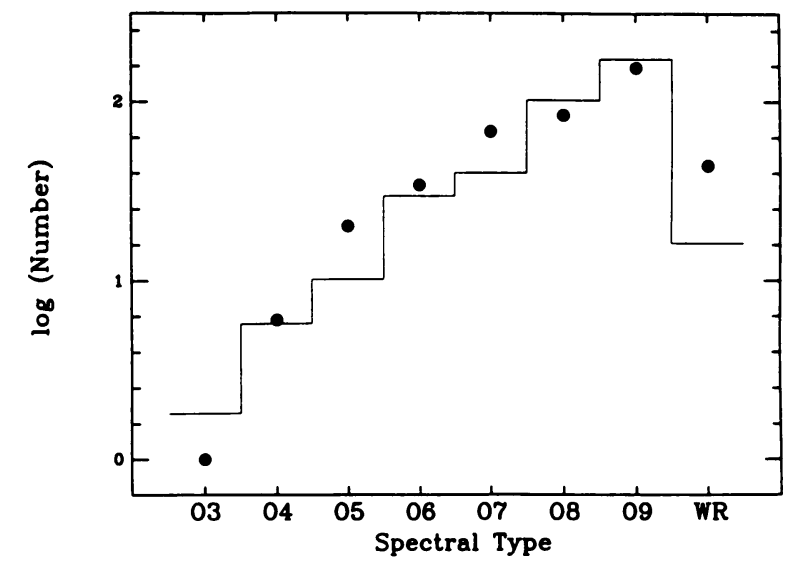

Figure 4. Observed (dots) and synthesized (histogram) number of O- and WR-stars in the solar neighborhood (from Leitherer 1990).

What are the reasons for this disagreement? On the observational side, I already discussed the fraction of $O$ stars which may still be hidden in primordial gas and dust. These stars are not included in Figure 4 and could reduce the discrepancy by $\sim 20 \%$. Humphreys and McElroy (1984) argue that the O-star sample in Figure 4 is severely incomplete for the latest types. This would also decrease the observed $\frac{W R}{O}$ ratio. Finally I would like to point out the sensitivity of the results to the adopted $T_{\text {eff }}$ versus spectral-type calibration (see Figure 
5). At the high-mass end, even slight changes to this calibration result in a significantly different relation between mass and spectral type. At the low-mass end ( $M \approx 20 M_{\odot}$ ), minor modifications to the adopted effective temperature of the coolest $O$ star (e.g., 09.5 or 09.7) significantly affects $\frac{W R}{O}$ since the number of late- $O$ stars vastly dominates the total number, given the effect of the inital mass function.

Figure 5. HRD with evolutionary tracks and Schmidt-Kaler's (1982) spectral-type calibration. Dots represent types $\mathrm{O} 3$ through $\mathrm{B} 7$ in steps of 1 subtype.

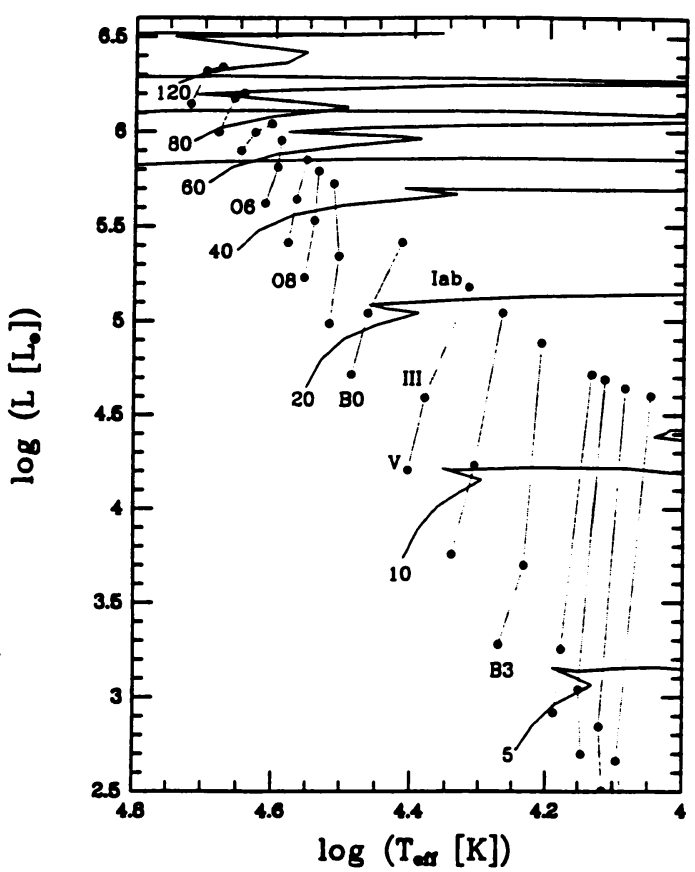

On the theoretical side, several uncertainties should be mentioned. Clearly, stellar evolution models themselves may undergo some revision (e.g., uncertainties in the adopted opacities, the amount of overshooting, and the mass-loss rates). The binary channel for the formation of WR stars has been neglected. Maeder (1990a) estimates that binary evolution may slightly increase the theoretical ratio of $\frac{W R}{O}$ but a significant effect should only be expected in a low-metallicity environment, such as the SMC. The models presented in Figure 4 assume a lower cut-off mass for the formation of WR stars of $33 M_{\odot}$. Lowering the cutoff mass will significantly increase the theoretical $\frac{W R}{O}$ ratio. As discussed in the previous section, the cut-off mass is still under debate, and values of $20 M_{\odot}$ or even lower are not incompatible with observations.

Given the uncertainties - both on the observational and on the theoretical side - I conclude that the observed relative numbers of WR stars and their progenitors in the solar neighborhood are basically in agreement with theoretical models and that they are consistent with the assumption that WR stars are bare cores left over from massive $O$ stars which lost their outer hydrogen-rich layers.

Recent theoretical models (Maeder 1990a) predict a very strong dependence of $\frac{W R}{O}$ on the initial chemical composition of the progenitor star. A lower metal content $Z$ leads to a significantly smaller value of $\frac{W R}{O}$. This is due to several consequences of lower $Z$ : the 
models predict smaller WR- and longer O-star lifetimes as well as higher cut-off masses for the formation of WR stars. Also, the initial mass of an $O$ star on the ZAMS decreases if $Z$ is lower. All these effects lead to a strong sensitivity of $\frac{W R}{O}$ on $Z$. In addition, the relative numbers of individual WR-subclasses, such as $\frac{W N}{W C}$ and $\frac{W C}{W R}$ are also expected to vary with $Z$.

It is difficult to test the variation of $\frac{W R}{O}$ with $Z$ in our Galaxy. The average galactocentric metallicity gradient derived from nebular analyses is $\sim 10^{-0.1} \mathrm{kpc}^{-1}$ (Talent and Dufour 1979). A factor-of-2 variation of $Z$ is reached only at a distance of $3 \mathrm{kpc}$. At such large distances, however, significant incompleteness for O stars sets in (Humphreys and McElroy 1984). It should be noted that Lennon et al. (1990) find no evidence for a galactocentric metallicity gradient within $3-4 k p c$ of the sun on the basis of quantitative spectroscopy of a sample of B stars. In fact, theory and observations do not agree too well with respect to the dependence of $\frac{W R}{O}$ on $Z$ if this test is performed in our galaxy (see Maeder, this conference).

In principle, a more promising way to study the influence of $Z$ comes from studying stellar statistics in Local Group galaxies which span a wide range of chemical composition. Unfortunately, the stellar census in these galaxies is still rather incomplete, and significant correction factors must be applied to the observations. Maeder, Lequeux, and Azzopardi (1980), Massey and Conti (1983), Freedman (1985), Azzopardi, Lequeux, and Maeder (1988), and Smith (1988) reach different conclusions regarding the dependence of the relative WR numbers to the numbers of their progenitors on $Z$. At present this is still an open issue but it is to be anticipated that more definitive conclusions can be drawn as soon as more complete statistics are available.

\section{Outlook: Massive Stars in Distant Galaxies}

Traditionally, constraints on stellar evolution in the upper HRD are almost entirely based on observational data collected in our Galaxy. Progress in telescope- and detector-technology makes it possible to study stellar evolution in galaxies at larger and larger distances. Some of these new data have already been discussed. Some galaxies of the Local Group are ideal laboratories to observe complete samples of massive stars at spatial and spectral resolution still high enough to obtain useful information. The ongoing work of Massey et al. (see, e.g., Massey, Parker, and Garmany 1989) in the Magellanic Clouds and of Moffat et al. (see, e.g., Moffat and Shara 1987) in M31/M33 provides important constraints for the theory of stellar evolution.

At even larger distances, galaxies can no longer be resolved into individual stars. Large populations of WR stars have been detected in the integrated spectra of some galaxies with active star formation (e.g., Kunth and Schild 1986). Although the detail of information is certainly degraded with respect to data from our Galaxy, such observations may well complement results derived locally. The number of stars contributing to the observed, integrated light is very large - one definite advantage over the number statistics in our Galaxy and irregular galaxies of the Local Group, which are mostly rather poor. Arnault, Kunth, and Schild (1989) published a grid of synthetic populations of massive stars with emphasis on the $\frac{W R}{O}$ ratio. If parameters such as, e.g., the initial mass function of massive stars can be constrained independently, observations of the stellar content of such galaxies may serve as an important tool to understand stellar evolution in the upper HRD. 


\section{References}

Arnault, P., Kunth, D., and Schild, H. 1989, Astr. Ap., 224, 73.

Arnett, W. D., Bahcall J. N., Kirshner, R. P., and Woosley, S. E. 1989, Ann. Rev. Astr. Ap., 27, 629.

Azzopardi, M., Lequeux, J., and Maeder, A. 1988, Astr. Ap., 189, 34.

Bertelli, G., Bressan, A., and Chiosi, C. 1984, Astr. Ap., 130, 279.

Blaha, C., and Humphreys, R. M. 1989, A. J., 98, 1598.

Bohannan, B., Abbott, D. C., Voels, S. A., and Hummer, D. G. 1986, Ap. J., 308, 728.

Chiosi, C. 1986, in IAU Symposium 116, Luminous Stars and Associations in Galaxies, ed. C. W. H. de Loore, A. J. Willis, and P. Laskarides (Dordrecht: Reidel), p. 317.

Chiosi, C., and Summa, C. 1970, Ap. Space Sci., 8, 478.

Conti, P. S., and Vacca, W. D. 1990, A. J., in press.

Conti, P. S., Garmany, C. D., de Loore, C. W. H., and Vanbeveren, D. 1983, Ap. J., 274, 302.

Doom, C. 1987, Astr. Ap., 182 , L43.

Drissen, L., Moffat, A. F. J., and Shara, M. M. 1990, Ap. J., in press.

Fitzpatrick, E. L., and Garmany, C. D. 1990, A. J., in press.

Freedman, W. L. 1985, A. J., 90, 2499.

Garmany, C. D. 1990, in Massive Stars in Starbursts, ed. T. Heckman, C. Leitherer, C. Norman, and N. Walborn (Cambridge: Cambridge University Press), in press.

Garmany, C. D., Conti, P. S., and Chiosi, C. 1982, Ap. J., 263, 277.

Garmany, C. D., Conti, P. S., and Massey, P. 1987, A. J., 93, 1070.

Hidayat, B., Admiranto, A. G., and van der Hucht, K. A. 1984, Ap. Space Sci., 99, 175.

Hillier, D. J. 1990, in Properties of Hot Luminous Stars, ed. C. D. Garmany (Provo:

Brigham Young University), p. 340.

van der Hucht, K. A., Hidayat, B., Admiranto, A. G., Supelli, K. R., and Doom, C. 1988, Astr. Ap., 199, 217.

Humphreys R. M. 1987, in Instabilities in Luminous Early-Type Stars, ed. H. J. G. L. M. Lamers, and C. W. H. de Loore (Dordrecht: Reidel), p.3

- . 1989, in IAU Colloquium 113, Physics of Luminous Blue Variables, ed. K.Davidson, A. F. J. Moffat, and H. J. G. L. M. Lamers (Dordrecht: Kluwer), p. 3.

Humphreys, R. M., and Davidson, K. 1979, Ap. J., 232, 409.

Humphreys, R. M., and McElroy, D. B. 1984, Ap. J., 284, 565.

Humphreys, R. M., Nichols, M., and Massey, P. 1985, A. J., 90, 101.

Kennicutt, R. C. 1983, Ap. J., 272, 54.

Kudritzki, R. P., Simon, K. P., and Hamann, W.-R. 1983, Astr. Ap., 118, 245.

Kudritzki, R. P., Gabler, A., Gabler, R., Groth, H. G., Pauldrach, A. W. A., and Puls, J. 1989, in IAU Colloquium 113, Physics of Luminous Blue Variables, ed. K.Davidson, A. F. J. Moffat, and H. J. G. L. M. Lamers (Dordrecht: Kluwer), p. 67.

Kunth, D., and Schild, H. 1986, Astr. Ap., 169, 71.

Lamers, H. J. G. L. M., Maeder, A., Schmutz, W., and Cassinelli, J. P. 1990, Ap. J., in press.

Langer, N., and El Eid, M. F. 1986, Astr. Ap., 167, 265.

Langer, N., El Eid, M. F., and Baraffe, I. 1990, Astr. Ap., in press.

Leitherer, C. 1990, Ap. J. Suppl., 73, 1.

Leitherer, C., Schmutz, W., Abbott, D. C., Hamann, W.-R., and Wessolowski, U. 1989, 
Ap. J., 346, 919.

Lennon, D. J., Kudritzki, R. P., Becker, S. R., Eber, F., Butler, K., and Groth, H. G. 1990, in Properties of Hot Luminous Stars, ed. C. D. Garmany (Provo: Brigham Young University), p. 315.

Lundström, I., and Stenholm, B. 1984, Astr. Ap. Suppl., 58, 163.

Maeder, A. 1983, Astr. Ap., 120, 113.

—. 1987, Astr. Ap., 173, 247.

- 1990a, Astr. Ap., in press.

- $1990 \mathrm{~b}$, Astr. Ap., in press.

Maeder, A., Lequeux, J., and Azzopardi, M. 1980, Astr. Ap., 90, L17.

Maeder, A., and Meynet, G. 1987, Astr. Ap., 182, 243.

—. 1988, Astr. Ap. Suppl., 76, 411.

Massey, P. 1981, Ap. J., 246, 153.

Massey, P., and Conti, P. S. 1983, Ap. J., 273, 576.

Massey, P., Parker, J. W., and Garmany, C. D. 1989, A. J., 98, 1305.

Mermilliod, J.-C., and Maeder, A. 1986, Astr. Ap., 158, 45.

Moffat, A. F. J., and Shara, M. M. 1987, Ap. J., 320, 266.

Schild, H., and Maeder, A. 1983, Astr. Ap., 127, 238.

-. 1984, Astr. Ap., 136, 237.

Schmidt-Kaler, T. 1982, in Landolt-Börnstein, New Series, Group VI, Vol. 2b, ed. K. Schaifers, and H. H. Voigt (Berlin: Springer), p. 1.

Schmutz, W., Hamann, W.-R., and Wessolowski, U. 1989, Astr. Ap., 210, 236.

Schmutz, W., Leitherer, C., Hubeny, I., Vogel, M., Hamann, W.-R., and Wessolowski, U. $1990, A p$. J., in press.

Schulte-Ladbeck, R. E. 1989, A. J., 97, 1471.

Stahl, O. 1986, Astr. Ap., 164, 321.

Smith, L. F. 1988, Ap. J., 327, 128.

St.-Louis, N., Drissen, L., Moffat, A. F. J., Bastien P., and Tapia, S. 1987, Ap. J., 322, 870.

Talent, D. L., and Dufour, R. J. 1979, Ap. J., 233, 888.

Walborn, N. R. 1976, Ap. J., 205, 419.

- 1988, in IAU Colloquium 108, Atmospheric Diagnostics of Stellar Evolution, ed. K. Nomoto (Berlin: Springer), p. 70.

- 1989, in IAU Colloquium 113, Physics of Luminous Blue Variables, ed. K. Davidson, A. F. J. Moffat, and H. J. G. L. M. Lamers (Dordrecht: Kluwer), p. 27.

- 1990, in IAU Symposium 148, The Magellanic Clouds, ed. R. F. Haynes, and D. K. Milne (Dordrecht: Kluwer), in press.

Wolf, B., and Zickgraf, F.-J. 1987 in Instabilities in Luminous Early-Type Stars, ed. H. J.

G. L. M. Lamers, and C. W. H. de Loore (Dordrecht: Reidel), p. 245

Wood, D. O. S., and Churchwell, E. 1989, Ap. J., 340, 265. 


\section{DISCUSSION}

De Greve: As a consequence of the interplay of stellar wind and overshooting, the evolutionary tracks show a rightward extension around $M_{b o l}=-8$ and a decrease in width above -9. How accurate can observations confirm this behavour in both regions?

Leitherer: One should be aware that this specific behaviour of the evolutionary tracks is a consequence of careful fine-tuning of the models by observations - rather than an independent prediction. Maeder and collaborators used a sample of young clusters to derive the amount of overshooting necessary to reproduce the observations. Therefore, the tracks will very closely resemble the observed main sequence width. However, it should be kept in mind that new results by Garmany et al. indicate that severe problems exist in the cluster membership of many OB stars. This will of course affect Maeder's results.

Lortet: A good place to look for ZAMS hot stars is the SMC where the dust content is low and we are able to see stars in less evolved regions. We found a few in Henize N 83 and 84 (the $\mathrm{K} 1$ region of the SMC).

Leitherer: It is indeed true that the SMC is an interesting place to study the ZAMS. Due to the low metal content of the SMC, theoretical models predict a significant shift of the ZAMS towards hotter temperatures. Unfortunately, we are still rather incomplete in our census of stars close to the ZAMS in the SMC (cf. the HRD of the LMC published by Fitzpatrick and Garmany). This work is still ongoing (mostly due to Conti and collaborators) and we will probably have to wait for a few more years to obtain statistically significant results.

Humphreys: Comparing the HRD's of the Galaxy and the LMC, you see a gradient in the number of stars stretching all the way from the hottest most luminous to the A-Type supergiants. This is not the same line as drawn by Fitzpatrick and Garmany. It is observed in the Galaxy, LMC and SMC and even in other Local Group galaxies, even though the data sets are much less complete. This is a question for the model-makers: What is the evolutionary origin of this gradient? Is it an artifact of how we are treating the data, due to observational selection or is there a physical explanation?

Sreenivasan: You make two observations, (1) that the Fitzpatrick-Garmany ridge in the LMC represents an ensemble of stars in different evolutionary phases, (2) the evolutionary origin of WR stars is determined entirely by their initial mass. I agree with the former and one possible explanation for it can be found in my poster outside but I disagree with the latter. A WR star phase is determined by two simultaneous processes, one that removes the outer layers and the other that transforms the core. The two related time-scales are determined not only by the initial mass but by other phenomena that determine stellar wind mechanisms and core growth.

Leitherer: I emphasized in my talk that the initial mass may not be the only parameter determining the late evolution of very massive stars. Other factors could be stellar rotation or the magnetic field. Cassinelli's models should be mentioned in this respect. I agree with you that it may be too naive to exclude $a$ priori parameters other than mass.

Wilson: What is the effect of obscuration by circumstellar dust shells on the observed distribution of luminosities for red supergiants?

Leitherer: Humphreys did several thorough studies to address this problem. We observe many red supergiants with massive dust shells. They are bright in the infrared where we can determine the bolometric luminosity. They are not above the Humphreys-Davidson limit. I should mention the case of M 101: If the canonical value for its distance modulus is taken then many red supergiants (and also blue supergiants) are above the H-D limit. The distance to $M 101$, however, is still under debate, and the stars may well be under the limit. 
Yungelson: (1) Comment: You cannot go with masses of WR progenitors as low as $\sim 15 M_{\odot}$, because stars of such a low mass do not have massive enough helium cores, predecessors of WR stars, that would produce helium stars hot enough to produce stellar winds able to give rise to WR phenomenon. (2) Question: When you are telling about deficiency of very young $\mathrm{O}$ stars relying on isochrones, do you make allowance for unresolved binarity, that can shift the ZAMS upwards?

Leitherer: (1) I agree with your comment. Actually, my mean low cut-off mass of $25 \pm 10 M_{\odot}$ does not conflict with your value. (2) No allowance for a shift of the ZAMS due to binarity has been made. This may decrease the problem of the observed lack of very young stars somewhat. However, I think we still need an additional explanation - like the ultracompact $H I I$ regions - to account for the entire effect.

Langer: (1) The diagonal iso-density lines in the observed HR diagram Humphreys referred to in her question, may well reflect the well known fact that blue loops from the Hayashiline extend to higher surface temperatures for higher masses (luminosities). (2) If this is the case ( $c f$. Fitzpatrick and Garmany, 1990), it would mean that almost all BSG's are post-RSG stars and should therefore be $N$-enriched, since blue loops are obtained only for tracks which ignite core helium burning as RSG's. This would be well in line of Walborn's $\mathrm{OBN} / \mathrm{OBC}$ interpretation.

Claus Leitherer

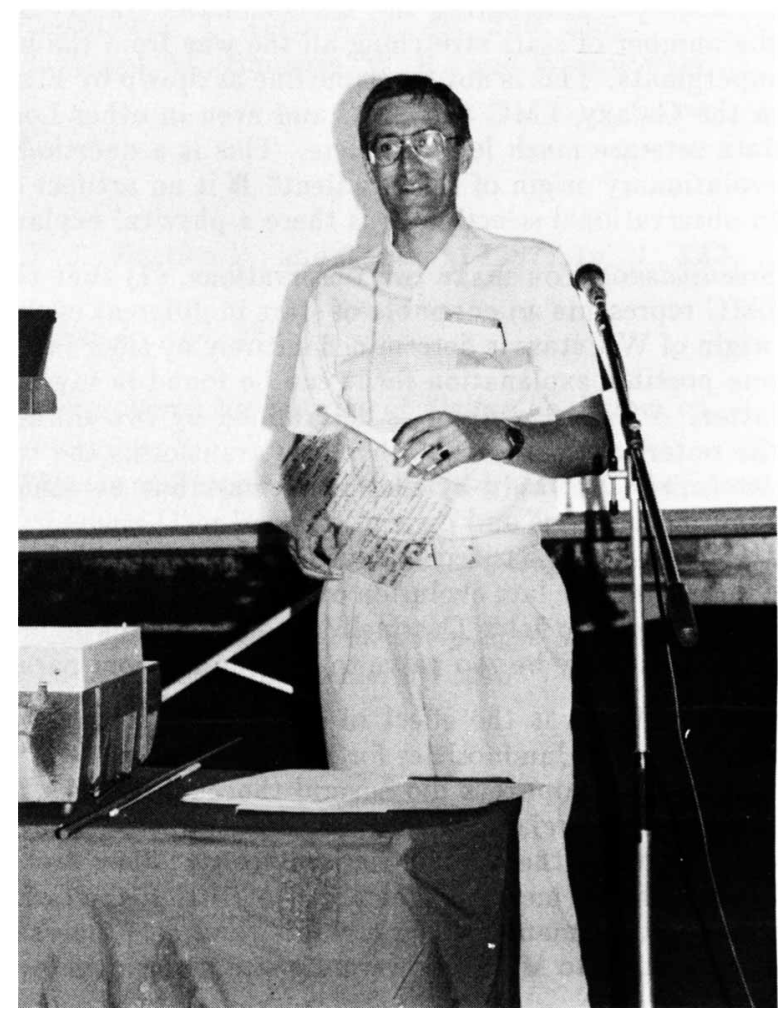

